\title{
A REPRESENTATION FORMULA FOR LINEAR VOLTERRA INTEGRAL EQUATIONS
}

\author{
BY J. M. BOWNDS AND J. M. CUSHING
}

Communicated by Hans Weinberger, November 1, 1972

ABSTRACT. It is shown that the solution to a finite system of linear Volterra integral equations may be expressed in terms of the fundamental solution to a so-called associated system of ordinary differential equations. The only requirement on the kernel is that it be expandable in a sufficiently convergent series of continuous matrices.

1. Introduction. The only goal here will be to establish a representation formula for the solution to the finite system of Volterra integral equations of the form

$$
u(x)=\varphi(x)+\int_{a}^{x} K(x, t) u(t) d t, \quad x_{0} \leqq a \leqq x<+\infty,
$$

in terms of the solution to a certain infinite system of ordinary differential equations. The applicability of the resultant representation formula may be varied, but we will show in future work how it leads to stability theorems for general integral equations. It may be pointed out here that the nature of the representation theorem to be proved is such that an explicit, closedform solution may be exhibited whenever such a solution can be found for, what will be called, the associated system of ordinary differential equations.

Let $K(x, t)$ be a continuous $k \times k$ matrix $(k<+\infty)$ on $x_{0} \leqq t \leqq x$ $<+\infty$, and $\varphi(x)$ be a continuous $k$-vector on $\left[x_{0},+\infty\right)$. In a previous work [1], we have shown that if $U(x, s)$ satisfies the $k \times k$ matrix equation

$$
U(x, s)=I+\int_{s}^{x} K(x, t) U(t, s) d t
$$

then the solution to (I) is given by

$$
u(x)=U(x, a) \varphi(a)+\int_{a}^{x} U(x, s) \varphi^{\prime}(s) d s,
$$

provided we make the additional assumption that $\varphi \in C^{1}\left[x_{0},+\infty\right)$. If $\partial U(x, s) / \partial s$ is continuous, it is easily shown that the alternate representation formula

$$
u(x)=\varphi(x)-\int_{a}^{x} \frac{\partial U}{\partial s}(x, s) \varphi(s) d s
$$

AMS (MOS) subject classifications (1970). Primary 45D05, 45M10; Secondary 45A02, 45A05, 45E99, 45F05, 45G05, 45G99, 45J05.

Key words and phrases. Volterra integral equations, representation formulas, infinite systems of ordinary differential equations. 
holds. These equations are, of course, analogous to the variation of constants formula in the theory of ordinary differential equations. These representations are related to others in the literature and the connection is made in [1]; see also [2]-[4].

2. The representation theorem. In another paper [5] concerning stability theory for (I), a special case of the theorem below was proved. In that work, it was assumed that the kernel is of Pincerele-Goursat type. We see here that this restriction may be replaced by the weaker assumption that $K(x, t)$ be expandable in a sufficiently convergent series of matrices

$$
K(x, t)=\sum_{n=1}^{\infty} A_{n}(x) B_{n}(t)
$$

Consider the following infinite system of ordinary differential equations which we call the associated system:

$$
\frac{d}{d x} \Phi_{m n}(x, t)=\sum_{k=1}^{\infty} B_{m}(x) A_{k}(x) \Phi_{k n}(x, t), \quad \Phi_{m n}(t, t)=\delta_{m n} I,
$$

for $x_{0} \leqq t \leqq x<+\infty$, where $\delta_{m n}$ is the Kronecker delta and $m, n=1,2, \ldots$. By a solution, we mean a double sequence of $k \times k$ matrices $\Phi_{m n}(x, t)$ which are continuously differentiable with respect to $x \geqq t$, satisfying (ADE). In the theorem below, it is assumed that (ADE) has a solution which, for fixed $x$ and $t$, is bounded uniformly in $m, n$. This assumption will be discussed later in the context of the theory of strong differential equations in a Banach algebra of infinite matrices. Let $\|\cdot\|$ be any norm on a $k \times k$ matrix.

TheOrem. Suppose that $K(x, t)$ satisfies $(\mathrm{K})$, where

$$
\alpha(x)=\sum_{n=1}^{\infty}\left\|A_{n}(x)\right\|, \quad \beta(x)=\sum_{n=1}^{\infty}\left\|B_{n}(x)\right\|
$$

define real-valued, integrable functions on every finite subinterval of $\left[x_{0},+\infty\right)$; and suppose that (ADE) has a solution $\Phi_{m n}(x, t)$ and that there exists a real-valued function $c(x, t)$ such that $\left\|\Phi_{m n}(x, t)\right\| \leqq c(x, t)<+\infty$, $x_{0} \leqq t \leqq x<+\infty, m, n=1,2, \ldots$, and $c(x, t) \beta(t)$ is integrable in $t$ on $\left[x_{0}, x\right]$. Then

$$
U(x, s)=I+\int_{s}^{x} \sum_{m=1}^{\infty} \sum_{n=1}^{\infty} A_{m}(x) \Phi_{m n}(x, t) B_{n}(t) d t,
$$

for $x_{0} \leqq s \leqq x<+\infty$. Here the summations and integral may be taken in any order.

PROOF. The convergence and the integrability of the integrand and the 
interchangeability of the integral and the sums follows from the inequality

$$
\sum_{m=1}^{\infty} \sum_{n=1}^{\infty}\left\|A_{m}(x) \Phi_{m n}(x, t) B_{n}(t)\right\| \leqq \alpha(x) c(x, t) \beta(t)<+\infty
$$

and the Lebesgue Monotone Convergence Theorem. Use has also been made here of the fact that a convergent, iterated double sum of positive terms may be summed in any manner and always to the same sum [9, p. 27]. We are, therefore, justified in substituting (RU) into the right-hand side of $(\mathrm{U})$, and we complete the proof by direct verification. The indicated substitution yields

$$
\begin{aligned}
I+\int_{s}^{x} \sum_{i=1}^{\infty} A_{i}(x) B_{i}(t)\left\{I+\int_{s}^{t} \sum_{m=1}^{\infty} \sum_{n=1}^{\infty} A_{m}(t) \Phi_{m n}(t, z) B_{n}(z) d z\right\} d t \\
=I+\int_{s}^{x} \sum_{i=1}^{\infty} A_{i}(x) B_{i}(t) d t \\
+\int_{t=s}^{x} \sum_{i=1}^{\infty} A_{i}(x) B_{i}(t) \int_{z=s}^{t} \sum_{m=1}^{\infty} \sum_{n=1}^{\infty} A_{m}(t) \Phi_{m n}(t, z) B_{n}(z) d z d t .
\end{aligned}
$$

From this point on we freely make use of the interchangeability of all the involved limits in this expression noting only that the justification is supplied in a manner as above. Consequently, after a commutation of the integral with respect to $z$ with the summation over $i$ and an application of Fubini's Theorem, we arrive at

$$
\int_{z=s}^{x} \sum_{i=1}^{\infty} \sum_{n=1}^{\infty} A_{i}(x)\left\{\int_{t=z}^{x} B_{i}(t) \sum_{m=1}^{\infty} A_{m}(t) \Phi_{m n}(t, z) d t\right\} B_{n}(z) d z
$$

for the second integral in (2.1). Also, it is easily seen that (ADE) is equivalent to the infinite system of integral equations

$$
\Phi_{i n}(x, z)=\delta_{i n} I+\int_{z}^{x} B_{i}(t) \sum_{m=1}^{\infty} A_{m}(t) \Phi_{m n}(t, z) d t .
$$

Hence, this integral simplifies to

$$
\sum_{i=1}^{\infty} \sum_{n=1}^{\infty} \int_{z=s}^{x} A_{i}(x) \Phi_{i n}(x, z) B_{n}(z) d z-\sum_{i=1}^{\infty} \int_{s}^{x} A_{i}(x) B_{i}(z) d z,
$$

which, upon substitution into (2.1), yields

$$
I+\sum_{i=1}^{\infty} \sum_{n=1}^{\infty} \int_{z=s}^{x} A_{i}(x) \Phi_{i n}(x, z) B_{n}(z) d z
$$

Since this expression is seen to be nothing more than $U(x, s)$ the proof is complete. 
3. On the associated differential equation. We briefly reconsider the assumption that (ADE) has a solution $\Phi_{m n}(x, t)$ which, at each $(x, t)$, has a bound $c(x, t)$ independent of $m$ and $n$. We note that (ADE) can be written as

$$
\begin{aligned}
d \Phi(x, t) / d x & =M(x) \Phi(x, t), \quad x_{0} \leqq t \leqq x, \\
\Phi(t, t) & =I,
\end{aligned}
$$

where $\Phi(x, t)=\left(\Phi_{m n}(x, t)\right), M(x)=\left(B_{m}(x) A_{n}(x)\right)$, and $I$ is the infinite identity matrix. If we introduce the Banach algebra $\mathscr{M}_{\infty}$ of infinite matrices with finite norm given by

$$
\|M\|_{\infty}=\sup _{i} \sum_{j=1}^{\infty}\left|m_{i j}\right| \text { or } \sup _{j} \sum_{i=1}^{\infty}\left|m_{i j}\right|
$$

for $M=\left(m_{i j}\right), m_{i j}$ real, then the usual existence theory for (ADE) applies when $M(x)$ is strongly continuous and we are guaranteed the existence of a strongly differentiable solution $\Phi(x, t)$ for all $x_{0} \leqq t \leqq x$. Moreover, in the theorem, $c(x, t)$ may be taken to be simply $K\|\Phi(x, t)\|$ (for any sufficiently large constant $K>0$ ) which is continuous in $t$. This is because the arbitrary matrix norm $\|\cdot\|$ is equivalent to the norm $\|M\|_{k}=\sup _{i} \sum_{j=1}^{k}\left|m_{i j}\right|$ and, hence, $\left\|\Phi_{m n}(x, t)\right\| \leqq K\left\|\Phi_{m n}(x, t)\right\|_{k} \leqq K\|\Phi(x, t)\|_{\infty}$ for some constant $K>0$. Consequently, all the hypotheses in the theorem concerning $\Phi_{m n}(x, t)$ and $c(x, t)$ are satisfied.

To remark on the strong continuity of $M(x)$ as defined above for (ADE), we note that the following simple hypothesis is easily shown to suffice:

Given any $x^{*} \in\left[x_{0},+\infty\right)$, there exists an $\left(L_{i j}\left(x^{*}\right)\right) \in \mathscr{M}_{\infty}$, such that to any $\varepsilon>0$ there exists a $\delta=\delta\left(\varepsilon, x^{*}\right)>0$ for which $\left|x-x^{*}\right| \leqq \delta$ implies $\left\|B_{i}(x) A_{j}(x)-B_{i}\left(x^{*}\right) A_{j}\left(x^{*}\right)\right\| \leqq \varepsilon L_{i j}\left(x^{*}\right)$.

The following corollary obtains from this observation.

Corollary. Suppose that $K(x, t), \alpha(x), \beta(x)$ are as in the Theorem and that $(\mathrm{H})$ holds. Then the representation $(\mathrm{RU})$ is valid.

We point out that if $K(x, t)$ can be represented as a finite sum in $(\mathrm{K})$ for continuous matrices $A_{n}, B_{n}$, then all of the hypotheses of this Corollary are valid. In this case (ADE) is a finite system of differential equations and the sums in (RU) are finite (see [5]). For example, if $K(x, t)$ is a scalar function for which $K(x, t)=a(x) b(t)$ for continuous scalar functions $a$ and $b$, then (ADE) reduces to a single, first-order equation, which, of course, can be integrated explicitly. Its solution is $\exp \left(\int_{t}^{x} b(z) a(z) d z\right)$ which, when substituted into (RU), yields the closed form solution

$$
U(x, s)=1+\int_{s}^{x} a(x) b(t) \exp \left(\int_{t}^{x} b(z) a(z) d z\right) d t
$$


for the fundamental matrix $U(x, s)$. Thus, the explicit solution

$$
u(x)=\varphi(x)+a(x) \int_{a}^{x} \varphi(s) b(s) \exp \left(\int_{s}^{x} b(z) a(z) d z\right) d s
$$

to the integral (I) results.

The possibility for other Banach algebra settings in the treatment of (ADE) and of the hypotheses of the theorem are, of course, quite plausible. This possibility will not be discussed here since the above norm is the most commonly and conveniently used.

\section{REFERENCES}

1. J. M. Bownds and J. M. Cushing, Some stability theorems for systems of Volterra integral equations, Applicable Anal. (to appear).

2. R. K. Miller, J. A. Nohel and J. S. W. Wong, A stability theorem for nonlinear mixed integral equations, J. Math. Anal. Appl. 25 (1969), 446-449. MR 38 \# 2556.

3. , Perturbations of Volterra integral equations, J. Math. Anal. Appl. 25 (1969), 676-690. MR 39 \#1920.

4. R. K. Miller, On the linearization of Volterra integral equations, J. Math. Anal. Appl. 23 (1968), 198-208.

5. J. M. Bownds and J. M. Cushing, Some stability criteria for linear systems of Volterra integral equations, Funkcial. Ekvac. 15(1972), 101-117.

6. E. Hille and R. S. Phillips, Functional analysis and semi-groups, rev. ed., Amer. Math. Soc. Colloq. Publ., vol. 31, Amer. Math. Soc., Providence, R.I., 1957. MR 19, 664.

7. E. Hille, Lectures on ordinary differential equations, Addison-Wesley, Reading, Mass., 1969. MR 40 \#2939.

8. - Pathology of infinite systems of linear first order differential equations with constant coefficients, Ann. Mat. Pura Appl. (4) 55 (1961), 133-148. MR 24 \# A3383.

9. E. Titchmarsh, Theory of functions, Oxford Univ. Press, London, 1939.

Department of Mathematics, University of Arizona, Tucson, Arizona 85721 